\title{
Microstrip circuit analog of a complex diffraction phenomenon
}

\author{
A. Fernández-Prieto, ${ }^{1}$ F. Medina, ${ }^{1, a)}$ and F. Mesa ${ }^{2}$ \\ ${ }^{1}$ Departamento de Electrónica y Electromagnetismo, Universidad de Sevilla, 41012-Sevilla, Spain \\ ${ }^{2}$ Departamento de Física Aplicada 1, Universidad de Sevilla, 41012-Sevilla, Spain
}

(Received 23 May 2009; accepted 21 June 2009; published online 14 July 2009)

\begin{abstract}
This letter presents a methodology to reproduce at microwave frequencies the physical behavior of certain diffraction structures that have been investigated in the optics community. The methodology will be exemplified with a microstrip circuit implementation of a transmission line system exhibiting an electromagnetic response that mimics the response of simple and compound diffraction gratings. The identification of such "bridge" circuit analogies could be very fruitful for the understanding and development of design strategies to devise practical components based on phenomena employed in the affine areas of optics and microwave engineering. (C) 2009 American Institute of Physics.
\end{abstract}

[DOI: 10.1063/1.3176436]

Extraordinary transmission (ET) of electromagnetic waves through 1D (one-dimensional) or two-dimensional periodic diffraction gratings has been a very popular research topic during the last ten years, since the discovery of the phenomenon $^{1}$ at the optical range. Even though ET was soon connected with periodicity, in a first stage, the plasmalike behavior of metals at optical frequencies (frequency dependent complex permittivity with large and negative real part) was considered essential for the phenomenon. In this regard, the excitation of surface plasmon polaritons (SPPs) was key to explain the unexpected frequency-selective enhanced transmission through otherwise almost opaque screens. However, ET peaks can also be observed at microwave or millimeter wave frequencies. ${ }^{2}$ At these frequencies, metals can no longer be regarded as plasmas, and therefore the specific constitutive relation of the metal should not play any essential role. Indeed, even perfect-conductor perforated screens exhibit enhanced transmission peaks. The SPP theory was accordingly extended to conform with these observations., The basic idea of that extension was that the structured metal surface supports certain surface-wave eigenmodes, which act as intermediary in the electromagnetic field enhancing process required for the appearance of transmission peaks (these waves have been called spoof plasmons or SPP-Bloch waves).

Recently some of the authors of this letter have developed an alternative equivalent theory to explain ET. Our theory does not explicitly rely on the excitation of SPP but on the perfect impedance matching condition. A comprehensive account of this point of view can be found in some recent papers that describe the nature of the model and its qualitative and quantitative predictions. ${ }^{5,6}$ Although the model involves components that are familiar to electrical engineers (transmission lines, waveguides, lumped capacitors, and inductors), it is not only a mere circuit analog but rather a different paradigm that can explain most of the ET systems previously studied on the basis of SPP, and also other ET systems where SPPs are not present (for instance, total transmission through small diaphragms inside closed waveguides ${ }^{5,7,8}$ ). This equivalent-circuit approach was later extended by some of the authors ${ }^{9}$ to explain the complex

${ }^{\text {a) }}$ Electronic mail: medina@us.es. transmission spectra observed in 1D compound gratings. Our proposed equivalent transmission line circuit reproduces all the qualitative and quantitative details of these optical system, which were previously studied both theoretically ${ }^{10,11}$ and experimentally. ${ }^{12-14}$ Starting from this circuit analogy, the present contribution will focus on the design of a microwave passive system that behaves in a manner totally analogous to that of single/compound gratings. More specifically, what we will propose in this paper is a simple microstrip circuit that directly implements the essential details of the transmission spectra observed in an optical compound grating. This work is expected to provide a useful approach to introduce some aspects of physical optics to microwave students or engineers that are not familiar with the topic. However, more important is that it provides a "bridge" methodology to go back and forth from optics to microwave engineering, thus paving the way to design devices based on phenomena known or observed in the complementary discipline.

A compound grating is a $1 \mathrm{D}$ infinite periodic array of groups of slits practiced on an opaque and electrically thick screen [see an example in Fig. 1(a)]. A planar transverse electromagnetic wave that normally impinges on the array is

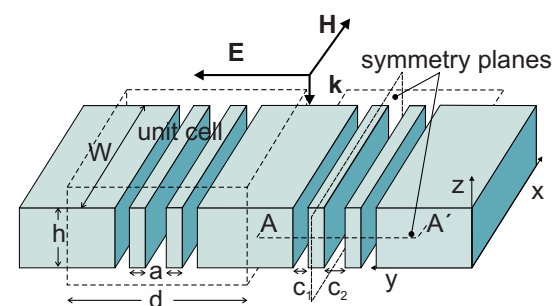

(a)

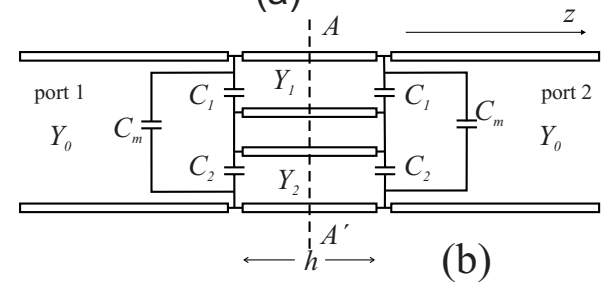

FIG. 1. (Color online) (a) Two cells of a compound grating having three slits per period of length $d$. The structure is electrically large along $x$ and $y$ directions. (b) Equivalent transmission line circuit following Refs. 9 and 15. 
reflected and transmitted. Due to the symmetry of the problem (geometry and excitation) as well as its periodic nature, the study of the complete structure can be reduced to the study of a unit cell of arbitrary width $W$ and vertical size $d$, such as that depicted in Fig. 1(a). Following the guidelines in Refs. 9 and 15, and provided that the wavelength of the impinging radiation is larger than the period of the structure, a transmission line problem can readily be identified with exactly the same scattering coefficients as those of the original diffraction problem. In particular, the transmission line circuit shown in Fig. 1(b) is the equivalent circuit for the three slits (per period) system in Fig. 1(a). The two transmission line sections with characteristic admittances $Y_{1}$ and $Y_{2}$ replace the slits. (Due to the symmetry of the unit cell only two different transmission lines are required to account for the three slits). The free space above and below the screen is replaced by the two transmission lines with characteristic admittance $Y_{0}$. In a typical situation $Y_{1}, Y_{2} \gg Y_{0}$ because $c_{1,2} \ll d$. Note that this same model is also valid for the case of four slits per period (using the appropriate values for $Y_{1}$ and $Y_{2}$ ). In general, $N$ transmission lines should be used for $2 N$ or $2 N-1$ slits per period.

As it is explained in Refs. 9 and 15, the compoundgrating structure behaves as a Fabry-Pérot system where the transmission-line resonators are capacitively loaded. In the case of one or two slits per period, a typical Fabry-Pérot transmission/reflection spectrum is observed. For wavelengths smaller than the period $d$, the situation is more involved because the impinging power will split into several grating lobes. However, even within the Fabry-Pérot regime and in the frequency range where a Fabry-Pérot resonance is expected for the case of a single slit per period, both theoretical $^{10,11}$ and experimental ${ }^{12-14}$ investigations show that the transmission spectrum presents profound dips when the number of slits per period is equal or larger than three. This fact was explained in ${ }^{10,11}$ using the concept of phase resonances. However, the equivalent circuit in Fig. 1(b) can be used to deduce in a simple manner why and where the transmission dips (zero transmission in ideal systems) must appear. Thus, in each Fabry-Pérot order, a number of total transmission peaks equal to the number of transmission lines with different electrical lengths involved in the model is obtained. The difference between the electrical lengths of the lines in Fig. 1(b) comes from the different loading capacitances. However this difference can also be achieved using different values of $h$ for each slit. A close inspection of the equations derived from the circuit model reveals that a transmission dip will always appear between each couple of total transmission peaks. For lossy structures, the transmission peaks will not reach total transmission. Additionally, and as expected from Kramers-Kronig relationships, the model also predicts sharp absorption peaks at the frequencies of the dips.

Our challenge now is to find a microstrip circuit that accounts for the main features of the transmission-line network shown in Fig. 1(b) [which is the circuit analog of the grating in Fig. 1(a)]. By inspection of the circuit in Fig. 1(b) we can see that the input and output transmission lines (characteristic admittance $Y_{0}$ ) are connected to a system of much lower characteristic impedance lines (lines with $Y_{1}$ and $Y_{2}$ ), which would be series connected if the capacitances were ignored. Our proposal for the physical microstriplike implementation of this system is the printed circuit shown in Fig. 2 . The characteristic impedance of the input and output trans-

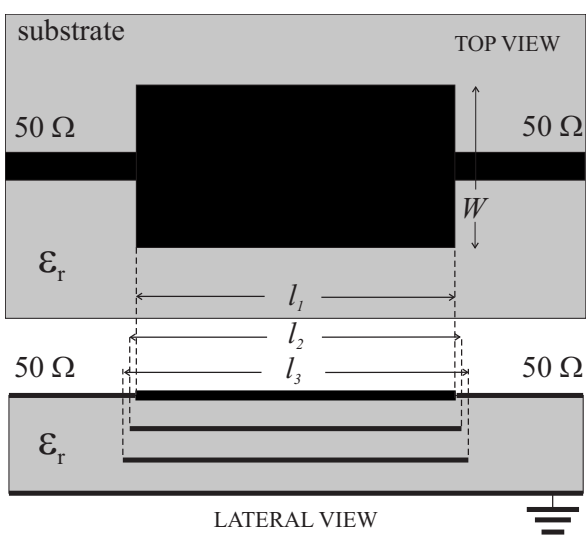

FIG. 2. Microstrip circuit that mimics compound gratings having five or six slits per period. For three or four slits per period one of the floating patches embedded in the substrate should be removed.

mission lines is $50 \Omega$ in order to match the measurement system. These lines are connected to a much wider microstrip (much lower characteristic impedance) in order to simulate the high characteristic impedance ratios involved in compound grating experiments. The number of floating conductor patches below the main resonator (the wide microstrip section at the top surface) should only be one to account for the circuit in Fig. 1(b) (which is valid for three or four slits per period). Two floating patches below the top resonator correspond to a circuit model such as that in Fig. 1(b) but with three intermediate transmission lines (instead of two). This case mimics the behavior of compound gratings having five or six slits per period. The multilayer printed circuit has been simulated (using the full-wave commercial software ADS MOMENTUM), fabricated, and measured. Since differences between the electrical lengths are essential to have transmission dips, we have chosen the lengths of the patches slightly different to make the effect more noticeable in the measured patterns. This is not required in the original compound grating because the electrical lengths of the various transmission lines involved in the problem are different enough due to differences between the edge capacitances.

In Fig. 3 it is shown the transmission spectra measured for two different multilayer structures. The figure shows two high transmission regions (corresponding to first and second order Fabry-Pérot resonances) where one or two transmission dips are clearly observed. This spectrum is qualitatively identical to the transmission spectra reported in previous theoretical and experimental works for compound gratings. ${ }^{11-14}$ In particular, when two transmission lines are involved in the microstrip circuit analog, a single transmission dip is observed. Two separate dips are obtained when three microstrip lines are present, what corresponds to the cases of five or six slits per period. This is exactly what happens in the compound grating problems and what our transmission line model predicts. From the measurement of the reflection coefficient we can obtain the power loss, which has also been plotted in Fig. 3. Note that strong absorption peaks are observed at the frequencies of the transmission dips (as it has been verified in Ref. 12 for gratings). Similar results have been found by the authors for other geometries and substrates. ${ }^{16}$

In brief, this letter has shown a simple microstrip circuit whose behavior is fully equivalent to the observed transmission behavior of compound 1D gratings studied by optics 

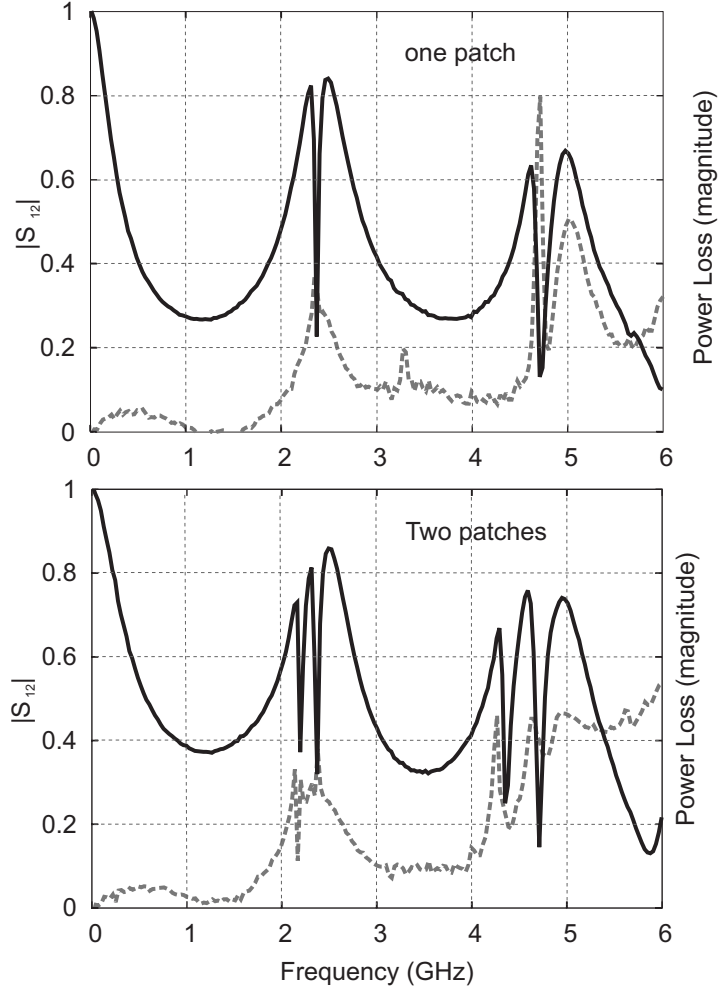

FIG. 3. Measured $\left|S_{12}\right|$ (black lines) of the microstrip circuit analog with one/two patches corresponding to compound 1D gratings with three(four)/ five(six) slits per period. Power loss is included (dashed gray lines). One dip per Fabry-Pérot resonance is found in the first case and two dips appear in the second case. Absorption peaks are found at the resonances. Parameters of the structure in Fig. 2: $W=30.25 \mathrm{~mm}, l_{1}=38.43 \mathrm{~mm}, l_{2}=42.0 \mathrm{~mm}$, $l_{3}=45.57 \mathrm{~mm}, h=0.49 \mathrm{~mm}, \varepsilon_{r}=2.43$, and $\tan \delta=0.002$.

researchers. This equivalence has been achieved after finding a transmission-line circuit analog of the phenomenon, and the implementation of this equivalent circuit at microwave frequencies. The experiments demonstrate that the circuitlike approach completely captures the physics of the transmission in these systems. The advantage is that a few parameters, several of which are known in closed form, are enough to characterize the whole reflection/absorption/transmission spectrum. Moreover, the rationale presented in this work is expected to be very fruitful in the design of experiments and/or devices in both disciplines: optics and microwaves. For instance, the electrical response observed in the case here treated of compound gratings with three/four slits per period can be seen as a notch filter of poor behavior. However, using a microwave engineering standpoint, one can envisage that if, instead of a single resonant stage, something similar to a Chebyshev transformer was used to increase the bandwidth of the pass band, the resulting optical structure could behave as a good notch filter.

This work has been supported by the Spanish Ministerio de Ciencia e Innovación (Project No. TEC2007-65376), Consolider Ingenio 2010 "Engineering Metamaterials" (Grant No. CSD2008-00066), and by Spanish Junta de Andalucía (Project No. TIC-253).

${ }^{1}$ T. W. Ebbesen, H. J. Lezec, H. F. Ghaemi, T. Thio, and P. A. Wolff, Nature (London) 391, 667 (1998).

${ }^{2}$ M. Beruete, M. Sorolla, I. Campillo, J. S. Dolado, L. Martín Moreno, and F. J. García-Vidal, IEEE Trans. Antennas Propag. 53, 1897 (2005).

${ }^{3}$ J. B. Pendry, L. Martín-Moreno, and F. J. García-Vidal, Science 305, 847 (2004).

${ }^{4}$ F. J. García de Abajo and J. J. Sáenz, Phys. Rev. Lett. 95, 233901 (2005).

${ }^{5}$ F. Medina, F. Mesa, and R. Marqués, IEEE Trans. Microwave Theory Tech. 56, 3108 (2008).

${ }^{6}$ R. Marqués, F. Mesa, L. Jelinek, and F. Medina, Opt. Express 17, 5571 (2009).

${ }^{7}$ R. Gordon, Phys. Rev. A 76, 053806 (2007).

${ }^{8}$ A. Kirilenko and A. Perov, IEEE Trans. Antennas Propag. 56, 3210 (2008).

${ }^{9}$ F. Medina, D. C. Skigin, and F. Mesa, Proceedings of the 38th European Microwave Conference (EuMC'08), Amsterdam, The Netherlands, 2008, p. 702 .

${ }^{10}$ D. C. Skigin and R. A. Depine, Phys. Rev. Lett. 95, 217402 (2005).

${ }^{11}$ D. C. Skigin and R. A. Depine, Phys. Rev. E 74, 046606 (2006).

${ }^{12}$ A. P. Hibbins, I. R. Hooper, M. J. Lockyear, and J. R. Sambles, Phys. Rev. Lett. 96, 257402 (2006).

${ }^{13}$ Y. G. Ma, X. S. Rao, G. F. Zhang, and C. K. Ong, Phys. Rev. B 76, 085413 (2007).

${ }^{14}$ M. Navarro-Cía, M. Beruete, D. C. Skigin, and M. Sorolla, Appl. Phys. Lett. 94, 091107 (2009).

${ }^{15}$ F. Medina, F. Mesa, and D. C. Skigin, "Extraordinary transmission through arrays of slits: A circuit theory model," IEEE Trans. Microwave Theory Tech. (to be published).

${ }^{16}$ F. Medina, A. Fernández-Prieto, and F. Mesa, Proceedings of the 39th European Microwave Conference (EuMC'09), Rome, Italy, 2009 (unpublished). 